Hugo Rojas ${ }^{1}$, Cristiane Ritter ${ }^{1}$, Felipe Dal Pizzol ${ }^{1}$

1. Laboratório de Fisiopatologia Experimental e Instituto Nacional de Ciência e Tecnologia Translacional em Medicina, Programa de PósGraduação em Ciências da Saúde, Unidade Acadêmica de Ciências da Saúde, Universidade do Extremo Sul Catarinense - Criciúma (SC), Brasil.

Conflitos de interesse: Nenhum.

Submetido em 3 de Fevereiro de 2011 Aceito em 17 de Maio de 2011

Autor correspondente:

Felipe Dal Pizzol

Laboratório de Fisiopatologia

Experimental, PPGCS, UNASAU

Universidade do Extremo Sul

Catarinense

CEP: 88806-000 - Criciúma (SC),

Brasil.

Fax: 55 (48) 34434817

E-mail: piz@unesc.net

\section{Mecanismos de disfunção da barreira hematoencefálica no paciente criticamente enfermo: ênfase no papel das metaloproteinases de matriz}

\author{
Mechanisms of dysfunction of the blood-brain barrier in critically \\ ill patients: emphasis on the role of matrix metalloproteinases
}

\section{RESUMO}

Será descrito a base fisiológica dos componentes da barreira hematoencefálica e suas propriedades. Além disto, pretende-se abordar o efeito particular das metaloproteinases e seu controle sobre as propriedades da matriz extracelular e a relação disto com disfunção da barreira hemotoencefálica. Finalmente se demonstrará o papel da metaloproteinases nas alteraçóes do sistema nervoso central em doenças associadas ao paciente criticamente enfermo.

Descritores: Barreira hematoencefálica; Proteína da matriz extracelular; Metaloproteinases da matriz

\section{INTRODUÇÁO}

O conceito de barreira hematoencefálica (BHE) surgiu no final do século XIX, na Alemanha, através de experimentos do cientista Paul Ehrlich onde, após injeções de corantes em animais tanto na circulação arterial como na venosa todos os órgãos, exceto o cérebro e a medula espinhal, se coravam, levando a hipótese de dois compartimentos. Além disto, Hugh Davson em sua revisão ${ }^{(1)}$ cita estudos de Bield e Kraus em 1898 e de Lewandowsky em 1900, onde atribuírem a ausência de efeitos farmacológicos no sistema nervoso central (SNC) quando os fármacos eram administradas de forma sistêmica. Anos depois, em 1913, Edwin Goldmann (um dos estudantes de Ehrlich) notou o fenômeno oposto injetando um corante diretamente no fluido cérebro espinhal, de animais, o qual manchou todo o SNC e nenhum dos órgãos periféricos. ${ }^{(2)}$ No entanto, somente nos anos 60 Reese e Karnovsky e Brightman e Reese repetiram as experiências de Ehrlich e Goldmann ao nível ultraestrutural, por meio da microscopia eletrônica, identificando os capilares do SNC e a célula endotelial como o sitio da barreira hematoencefálica. ${ }^{(1)}$

\section{Barreira hematoencefálica (BHE)}

\section{Descrição geral da BHE}

O sistema nervoso central (SNC) é uma zona privilegiada, que normalmente é protegido por três elementos estruturais: a BHE com a interface entre o encéfalo e os vasos sanguíneos; a barreira sangue - liquido cefalorraquidiano (BSLCR), formada pelo plexo coróide e a membrana aracnóide com os vasos sanguíneos e o líquido cefalorraquidiano; e a barreira e sangue - aracnóide (BSA), que é a interface dos vasos sanguíneos com a camada do epitélio da aracnóide subjacente a dura-máter das meninges. ${ }^{(3)}$ Estas barreiras são estruturas especializadas do SNC que controlam 
e regulam a homeostase do cérebro, medula espinhal, liquido cefalorraquidiano com o resto dos sistemas. ${ }^{(4,5)}$

\section{Componentes da BHE}

\section{- Células endoteliais}

A barreira é formada pela presença das junçóes endoteliais que controlam a abertura e fechamento coordenada das junçóes célula-célula. ${ }^{(6)}$ Estas junçóes são compostas de diferentes complexos multi-proteícos, como as junçôes apertadas (JA) e as junçóes endoteliais aderentes (JEA) que são os principais reguladores da permeabilidade celular. ${ }^{(7)}$ As JA consistem em três proteínas integrais de membrana denominadas claudina, ocludina e moléculas de adesão juncional e um número de proteínas citoplasmáticas acessórias, incluindo a zonula occludens (ZO) ZO-1, ZO-2 e ZO- 3, cingulina e outras proteínas guanilato quinase associadas a membrana (MAGUK). Estas proteínas acessórias conectam as proteínas de membrana à actina para a manutenção da integridade estrutural e funcional do endotélio. As JEA possuem proteínas de membrana chamadas caderinas, que se unem com a actina via proteínas intermediárias, denominadas cateninas $(\alpha, \beta, \mathcal{X})$, para formar contatos adesivos intercelular e interatuar com as JA. As células endoteliais cerebrais (CEC) são apoiadas sobre uma lamina basal que contem moléculas da matriz extracelular cobrindo mais de $90 \%$ da superfície das células endoteliais, também estando envolvida na permeabilidade da BHE. ${ }^{(8,9)}$

\section{- Ocludinas}

Foram as primeiras proteínas integrais de membrana a serem identificadas nas JA das células endoteliais. A ocludina é uma fosfoproteína de $65 \mathrm{kDa}$ com 4 domínios transmembrana, ligados a duas porções extracelulares ricas em tirosina no domínio intracelular carboxi e amino terminal. Sua função deve ser vista mais em um contexto regulador do que como uma proteína estrutural importante no estabelecimento das propriedades da barreira. Seu domínio citoplasmático carboxi terminal fornece a conexão da ocludina com o citoesqueleto assegurando a alta resistência elétrica das monocamadas das células endoteliais e diminuiçáo da permeabilidade paracelular. ${ }^{(10)}$

\section{- Claudinas}

As claudinas sáo proteínas integrais, de $21-28 \mathrm{kDa}$ com quatro domínios transmembrana, duas porçóes extracelulares e domínios citoplasmáticos carboxi e amino terminal com uma porção intracelular curta. ${ }^{(11,12)}$ As porçôes extracelulares se interligam com claudinas adjacentes das células endoteliais, formando a vedação primária das
JAs. ${ }^{(11)} \mathrm{O}$ principal papel das claudinas é a regulação da seletividade paracelular aos íons pequenos. Para isto as interaçóes entre as diferentes classes de interagem de duas maneiras: lateralmente no plano da membrana (interaçóes heteroméricos) ou a ligação cabeça a cabeça entre células adjacentes (interaçóes heterotípica). ${ }^{(13,14)}$

\section{- Astrócitos}

No cérebro humano, os astrócitos são as células gliais em forma de estrela que geralmente superam o número de neurônios por dez vezes, ${ }^{(15)}$ cujos prolongamentos finais formam um rendilhado de lamelas próximo e aderido na superfície externa do endotélio da BHE e a membrana basal respectiva. ${ }^{(16)}$ Um crescente número de resultados in vitro e in vivo suportam a idéia de que os astrócitos são células excitáveis e desempenham um papel importante no processamento de informaçóes e modulação da atividade neuronal. ${ }^{(17)}$ São importantes no controle do tônus vascular, ${ }^{(15)}$ neste sentido o papel dos astrócitos na manutenção e sinalização das células endoteliais da microvasculatura cerebral tem sido estudadas na gênese de diferentes doenças e na integridade da BHE. ${ }^{(18)}$

\section{- Pericito}

O recrutamento e a interação dos pericitos com o endotélio é essencial para a formação, maturação e manutenção da BHE. Os pericitos comunicam-se diretamente com às células endoteliais através de invaginaçóes referidas como "peg socket" onde um único pericito pode estar em contato com várias células endoteliais, permitindo uma camada adicional de comunicação ${ }^{(19)}$ e de estabilidade mecânica dos vasos. Em estudos recentes têm-se mostrado que pericitos são células cerebrais contráteis que regulam o fluxo sanguíneo capilar desempenhando um papel importante na manutençáo da BHE com a auto-regulação e homeostase cerebral.

Tem-se determinado em estudos in vivo que as interaçóes de pericitos com a célula endotelial são críticos para regular a BHE e a ruptura dessas interaçóes pode levar a disfunção desta barreira e a neuroinflamação. ${ }^{(20)}$ Além disto, estudos in vitro demonstram que os pericitos expressam moléculas como o fator de crescimento endotelial vascular (VEFG) e metaloproteinases de matriz (MMPs) que regulam a integridade da BHE. ${ }^{(21)}$

\section{- Matriz extracelular (MEC)}

O espaço extracelular representa aproximadamente $20 \%$ do volume total do cérebro sendo preenchido com uma matriz extracelular (MEC) altamente organizada, localizada na interface entre os vasos sanguíneos e a glia. A MEC parece servir como uma âncora com o endotélio através das interaçóes de proteínas da MEC com os receptores endoteliais 
tipo integrinas ${ }^{(7)}$ que desempenham um papel fundamental na sinalizaçáo celular, migraçáo e formaçáo capilar cerebral durante a angiogênese. ${ }^{(9)}$ Tais interaçōes célula-matriz podem estimular uma série de vias de sinalização intracelular nas células vasculares, neurônios e células gliais de apoio, alem de serem essenciais na BHE por que possivelmente participe na manutenção das proteínas endoteliais nas JA. ${ }^{(7,16,22)}$

A quebra da MEC pode levar a alteraçóes no citoesqueleto das células endoteliais da microvasculatura cerebral, que por sua vez afeta as proteínas das JA e a integridade da barreiracom o aumento da permeabilidade desta em estados patológicos. ${ }^{(16)}$

\section{Funçóes da barreira hematoencefálica}

A BHE é um importante componente da rede de comunicação que conecta o sistema nervoso central e os tecidos periféricos, alem disso funciona como uma interface que limita e regula a troca de substâncias entre sangue e o sistema nervoso central. ${ }^{(23)}$

A impermeabilidade da BHE é o resultado de uma série de características únicas, que acrescenta dificuldade à moléculas tentando penetrar nesta barreira. Esta propriedade é baseada na existência de uma permeabilidade muito restrita do endotélio, além da presença de enzimas degradantes presentes em grande número no interior do endotélio de modo que, com exceção de água, gases como oxigênio e o dióxido de carbono e determinadas moléculas lipossolúveis muito pequenas podem passar de forma íntegra. ${ }^{(23)}$ As moléculas hidrofílicas, que são essenciais para o metabolismo do cérebro, tais como íons, glicose, aminoácidos e componentes de ácido nucléico, passam pela BHE através de canais especializados. ${ }^{(24)}$ Já o transporte de moléculas hidrofílicas, tais como peptídeos e proteínas que não têm um sistema de transporte específico é muito mais lento do que as moléculas lipofílicas, no entanto, as quantidades que atravessam a BHE podem ser suficientes para causar um efeito mediado por receptores nos neurônios. Alguns tipos especiais de proteínas ou peptídeos como, por exemplo, hormônios periféricos e peptídeos regulatórios que exercem sua ação no cérebro geralmente têm sistemas especializados de transporte saturável em toda a BHE. Desta forma a BHE se torna altamente restritiva, mas de qualquer forma pode ser incapaz de impedir a passagem de alguns toxinas e agentes terapêuticos da corrente sanguínea para o cérebro. ${ }^{(24,25)}$

Além das funçóes de permeabilidade seletivaa BHE possui aspectos importantes como funçóes neuroimune, incluindo a secreção de citocinas, prostaglandinas e óxido nítrico. A BHE pode receber o estimulo de um compartimento (p.ex. o sistêmico) e, simultaneamente, responder com secreções para o outro (p.ex. o sistema nervoso central), sendo esta função de papel central na resposta neuroimune. Por exemplo, LPS aplicado na superfície das células do cérebro abluminal endotelial estimula a secreção de IL-6 a partir de sua superfície luminal. ${ }^{(25)}$

\section{Metaloproteinases de matriz (MMP)}

As metaloproteinases de matriz extracelular são enzimas, normalmente encontradas em sua forma inativa, da família de proteases dependentes de zinco sendo, até o momento, encontrados 23 membros em humanos. ${ }^{(26)}$ Estas proteínas são classificadas em: a) colagenases verdadeiras (MMP: 1, 8,13), que digerem a tripla hélice do colágeno, b) gelatinases (MMP: 2, 9), que atingem o colágeno e a gelatina desnaturada, c) estromelisinas (MMP: 3,10,11) que degradam as proteoglicanas, d) matrilisinas (MMP: 7, 26) que degradam proteoglicanas, fibronectina e laminina, e) metaloproteinase ligada à membrana MMP-MT (MMP: $14,15,16,17,23,24,25)$ degradam além da gelatina, fibronectina e agrecan e outros substratos de MEC, f) outras MMP (MMP:12,19,20,21,27 e 28) (27) capazes de degradar todos os componentes da MEC e proteínas de tecido conjuntivo. ${ }^{(28)}$

Os efeitos das MMP são rigidamente controlados por três mecanismos: (1) regulação da transcrição de genes, (2) regulação da ativação da pró-enzima e (3) presença de inibidores específicos (TIMP). ${ }^{(27)} \mathrm{O}$ controle da expressão gênica das MMP é dado em resposta a estímulos de citocinas (IL 1,4,6 e TNF $\alpha$ ), espécies reativas de oxigênio (ROS), oxido nítrico (NO), hormônios e fatores de crescimento, que se ligam a receptores na superfície da célula, desencadeando cascatas de sinalização intracelulares que, por sua vez, ativam fatores de transcrição que se ligam à regiōes responsivas presentes no promotor de genes de diferentes MMP. ${ }^{(29)}$ Por outro lado, as MMP são normalmente localizadas no citosol em um estado inativos e podem ser clivadas por proteases, como a plasmina, o ativador do plasminogênio tecidual (tPA) ou outras MMP ao seu estado ativo. ${ }^{(30)}$ Os inibidores teciduais endógenos de metaloproteinases (TIMP) são os principais inibidores fisiológicos das MMP, além disto a $\alpha 2$ - macroglobulina presentes no soro também pode inibir a atividade das MMP. Os TIMP são um conjunto de proteínas que são capazes de inibir as MMP solúveis através das interaçóes com uma razão estequiométrica 1:1, formando complexos TIMP-MMP que tornam a protease incapaz de se ligar ao substrato. Atualmente se conhecem quatro membros das famílias TIMP e as MMP podem ser inibidas por um número diferente deles. ${ }^{(30)}$ Cada TIMP tem características únicas; TIMP-1, TIMP-2 e TIMP- 4 
estão presentes na forma solúvel e TIMP-3 está fortemente vinculado ao $\mathrm{MEC}^{(31)}$ estando estes inibidores relacionados a promoção do crescimento celular, plasticidade sináptica e atividades antiangiogênicas e antiapoptoticas. ${ }^{(32)}$

\section{Disfunçáo da barreira hematoencefálica}

A disfunção da BHE, muitas vezes chamado de "abertura da BHE", tem sido descrita há muito tempo como um elemento-chave da progressão de várias doenças do SNC. ${ }^{(33)}$ Provavelmente isto esteja relacionado a exposição do microambiente cerebral a substâncias potencialmente nocivas, podendo resultar em uma perda da homeostase, comprometimento da oferta de sinalização neuronal e morte celular.

O papel central das MMPs na degradação da matriz neurovascular e a gênese de diferentes processos patológicos têm sido demonstrados em vários modelos de neuroinflamação. Como a regulação e ativação da expressão das MMP são complexas e bem controladas a perda desse controle se encontra envolvido na fisiopatologia da quebra da BHE. Desta forma se permite que circulem substâncias neurotóxicas para o parênquima cerebral, com a infiltração de leucócitos e ativação da microglia, promovendo resposta inflamatória que é encontrada em diferentes patologias do doente crítico como o acidente vascular encefálico (AVE) e traumatismo crânio-encefálico (TCE). ${ }^{(16,34)}$

Estudos em animais submetidos a modelo de AVE se observa uma ativação de MMP, levando a degradação da lâmina basal e da MEC com a quebra da BHE. Isto leva a aumento de fragilidade dos vasos de pequeno calibre contribuindo a transformação hemorrágica após o AVE isquêmico. Além disto, se encontrou uma ativação de MMP-2 na isquemia cerebral transitória nas primeiras etapas da lesão e conseqüente degradação da claudina (parte estrutural da JA e conseqüente quebra da BHE). A MMP-9 parece também ser relevante na fase aguda nas áreas peri-infarto por ter relação com a infiltração de neutrófilos ao redor dos capilares com piora da morte neuronal associada ao AVE. ${ }^{(30,34)}$ Embora as investigações se concentrem nas funçôes das MMP-2 e MMP-9, outros membros podem desempenhar papéis importantes. Por exemplo, a MMP-3 pode ser ativada após isquemia / reperfusáo no cérebro de ratos causando a ruptura da MEC e contribuindo para a abertura da BHE levando a neuroinflamação. Finalmente, MMP-13 foi recentemente encontrada no cérebro de ratos após isquemia sendo localizadas no núcleo das células neuronais e vasos do hipocampo tendo uma relaçáo da ruptura da BHE no AVE. ${ }^{(35,36)}$ Estas alteraçóes também podem ser relevantes no sangramento cerebral associado ao uso de trombolítiocos. Estes agentes terapêuticos são associados a ativação de MMP e em modelos animais a inibição farmacológica de MMP foi capaz de reduzir o volume da hemorragia cerebral associada ao t-PA. ${ }^{(37,38)}$

O estudo post-mortem em humanos valida que a MMP-9 está aumentada no tecido cerebral na zona do infarto e periinfarto dos pacientes com AVC. ${ }^{(39)}$ Em um estudo recente também em humanos, se observou uma associação entre os níveis plasmáticos de MMP-9 e rompimento da BHE após acidente vascular cerebral. ${ }^{(40)}$ Tomados em conjunto, o acúmulo de dados experimentais e clínicos sugerem que as MMP podem ser um biomarcador na lesão neurovascular durante a fase aguda do AVE e sugerem o uso de t-PA em conjunto com inibidor de MMP nos pacientes eletivos para trombólise. ${ }^{(37-41)}$

Em estudos animais se demonstrou que em modelo de TCE houve aumento nos níveis de MMP-9 em regióes corticais e hipocampais. A MMP-2 aumenta neste modelo dias após o TCE, sugerindo uma cascata de ativação de MMP onde a rápida ativação de MMP-9 ${ }^{(42)}$ pode estar envolvida na ativação sustentada de MMP-2. ${ }^{(43)}$ Estas alteraçóes estáo associadas temporal e espacialmente com a ruptura da BHE e a formação de edema cerebral, e o uso de inibidores de MMP leva a redução da área da lesão e da extravasão de água no cérebro. Camundongos transgênicos para MMP-9 apresentavam tamanhos significativamente menores de lesão em comparação com os camundongos de tipo selvagem em modelo de TCE. Além de MMP2 e 9 também é descrita MMP-3 em modelo animal de TCE levando ao edema vasogênico e conseqüente a morte celular. ${ }^{(44)}$ Estudos em humanos encontraram níveis elevados de MMP-2 e MMP-9 no plasma e no cérebro na fase inicial do TCE, ${ }^{(45)}$ além de uma estreita relação entre citocinas e aumento das MMPs na fase aguda. ${ }^{(36,41,46-50)}$

Em estudos em animais com indução de status epileticus foi determinado o aumento da MMP-2 no hipocampo. (51,52) Em soro de crianças com status epileticus, os níveis de MMP-9 foram significativamente maiores e o inibidor desta TIMP-1 foi significativamente menor quando comparados ao controle o que pode induzir a disfunção da BHE e o edema cerebral. ${ }^{(53)}$

\section{COMENTÁRIOS FINAIS}

Avanços na neurobiologia molecular estão rapidamente levando a uma compreensão melhor das alteraçóes agudas no SNCem diferentes doenças associadas ao paciente crítico. Nosso grande desafio é determinar com precisão em que doenças do paciente crítico as alteraçóes de MMP sáo relevantes para a disfunçáo do SNC e de que forma intervençóes terapêuticas sobre estas enzimas podem contribuir para estabilizar a BHE, atenuando a disfunção neurológica. 


\section{ABSTRACT}

This paper aims to describe the physiological basis of the blood-brain barrier components and its properties. Additionally, the particular effects of metalloproteinases and their control over the extracellular matrix and its relationship with blood-brain barrier dysfunction are discussed. Finally, the role of metalloproteinases on changes in the central nervous system in critically ill patients is discussed.

Keywords: Blood-brain barrier; Extracellular matrix protein; Matrix metalloproteinases

\section{REFERÊNCIAS}

1. Davson H. Review lecture. The blood-brain barrier. J Physiol. 1976;255(1):1-28.

2. Wiesendanger M. Constantin von Monakow (1853-1930) and Lina Stern (1878-1968): early explorations of the plexus choroideus and the blood-brain-barrier. Schweizer Archiv für Neurologie und Psychiatrie. 2010;161(4):140-4.

3. Correale J, Villa A. Cellular elements of the blood-brain barrier. Neurochem Res. 2009;34(12):2067-77.

4. Banks WA. Characteristics of compounds that cross the blood-brain barrier. BMC Neurol. 2009;9 Suppl 1:S3.

5. Hawkins BT, Davis TP. The blood-brain barrier/ neurovascular unit in health and disease. Pharmacol Rev. 2005;57(2):173-85

6. Stamatovic SM, Keep RF, Andjelkovic AV. Brain endothelial cell-cell junctions: how to "open" the blood brain barrier. Curr Neuropharmacol. 2008;6(3):179-92.

7. Bazzoni G, Dejana E. Endothelial cell-to-cell junctions: molecular organization and role in vascular homeostasis. Physiol Rev. 2004;84(3):869-901. Review.

8. Bauer H, Zweimueller-Mayer J, Steinbacher P, Lametschwandtner A, Bauer HC. The dual role of zonula occludens ( $\mathrm{ZO})$ proteins. J Biomed Biotechnol. 2010;2010:402593.

9. Zlokovic BV. The blood-brain barrier in health and chronic neurodegenerative disorders. Neuron. 2008; 57(2):178-201.

10. Persidsky Y, Ramirez SH, Haorah J, Kanmogne GD. Bloodbrain barrier: structural components and function under physiologic and pathologic conditions. J Neuroimmune Pharmacol. 2006;1(3):223-36. Review.

11. Lal-Nag M, Morin PJ. The claudins. Genome Biol. 2009; $10(8): 235$.

12. Furuse M. Molecular basis of the core structure of tight junctions. Cold Spring Harb Perspect Biol. 2010;2(1):a002907.

13. Findley MK, Koval M. Regulation and roles for claudin-family tight junction proteins. IUBMB Life. 2009;61(4):431-7.

14. Ohtsuki S, Sato S, Yamaguchi H, Kamoi M, Asashima T, Terasaki T. Exogenous expression of claudin-5 induces barrier properties in cultured rat brain capillary endothelial cells. J Cell Physiol. 2007;210(1):81-6.

15. Giaume C, Koulakoff A, Roux, L, Holcman D, Rouach N. Astroglial networks: a step further in neuroglial and gliovascular interactions. Nat Rev Neurosci. 2010;11(2): 87-99.

16. Cardoso FL, Brites D, Brito MA. Looking at the bloodbrain barrier: molecular anatomy and possible investigation approaches. Brain Res Rev. 2010;64(2):328-63.

17. Pereira A Jr, Furlan FA. Astrocytes and human cognition: modeling information integration and modulation of neuronal activity. Prog Neurobiol. 2010;92(3):405-20.

18. Willis CL, Nolan CC, Reith SN, Lister T, Prior MJ, Guerin $\mathrm{CJ}$, et al. Focal astrocyte loss is followed by microvascular damage, with subsequent repair of the blood-brain barrier in the apparent absence of direct astrocytic contact. Glia. 2004;45(4):325-37.

19. Armulik A, Abramsson A, Betsholtz C. Endothelial/pericyte interactions. Circ Res. 2005;97(6):512-23. Review.

20. Daneman R, Zhou L, Kebede AA, Barres BA. Pericytes are required for blood-brain barrier integrity during embryogenesis. Nature. 2010;468(7323):562-6.

21. Thanabalasundaram G, Pieper C, Lischper M, Galla HJ. Regulation of the blood-brain barrier integrity by pericytes via matrix metalloproteinases mediated activation of vascular endothelial growth factor in vitro. Brain Res. 2010;1347:1-10

22. Zimmermann DR, Dours-Zimmermann MT. Extracellular matrix of the central nervous system: from neglect to challenge. Histochem Cell Biol. 2008;130(4):635-53.

23. Banks WA. Blood-brain barrier as a regulatory interface. Forum Nutr. 2010;63:102-10. Review.

24. Begley DJ, Brightman MW. Structural and functional aspects of the blood-brain barrier. Prog Drug Res. 2003;61:39-78.

25. Banks WA, Erickson MA. The blood-brain barrier and immune function and dysfunction. Neurobiol Dis. 2010;37(1):26-32.

26. Zhang H, Adwanikar H, Werb Z, Noble-Haeusslein LJ. Matrix metalloproteinases and neurotrauma: evolving roles in injury and reparative processes. Neuroscientist. 2010;16(2):156-70.

27. Wright JW, Harding JW. Contributions of matrix metalloproteinases to neural plasticity, habituation, associative learning and drug addiction. Neural Plast. 2009;2009:579382.

28. Kupai K, Szucs G, Cseh S, Hajdu I, Csonka C, Csont T, Ferdinandy P. Matrix metalloproteinase activity assays: Importance of zymography. J Pharmacol Toxicol Methods. 
2010;61(2):205-9.

29. Sun J. Matrix metalloproteinases and tissue inhibitor of metalloproteinases are essential for the inflammatory response in cancer cells. J Signal Transuct. 2010;2010. pii: 985132.

30. Wang J. Preclinical and clinical research on inflammation after intracerebral hemorrhage. Prog Neurobiol. 2010;92(4):463-77.

31. Sekhon BS. Matrix metalloproteinases - an overview. Res Rep Biol. 2010;(1):1-20.

32. Brew $K$, Nagase $H$. The tissue inhibitors of metalloproteinases (TIMPs): an ancient family with structural and functional diversity. Biochim Biophys Acta. 2010;1803(1):55-71.

33. Weiss N, Miller F, Cazaubon S, Couraud PO. The bloodbrain barrier in brain homeostasis and neurological diseases. Biochim Biophys Acta. 2009;1788(4):842-57.

34. Candelario-Jalil E, Yang Y, Rosenberg GA. Diverse roles of matrix metalloproteinases and tissue inhibitors of metalloproteinases in neuroinflammation and cerebral ischemia. Neuroscience. 2009;158(3):983-94.

35. Solé S, Petegnief V, Gorina R, Chamorro A, Planas AM. Activation of matrix metalloproteinase- 3 and agrin cleavage in cerebral ischemia/reperfusion. J Neuropathol Exp Neurol. 2004;63(4):338-49.

36. Rosell A, Alvarez-Sabín J, Arenillas JF, Rovira A, Delgado P, Fernández-Cadenas I, et al. A matrix metalloproteinase protein array reveals a strong relation between MMP-9 and MMP-13 with diffusion-weighted image lesion increase in human stroke. Stroke. 2005;36(7):1415-20.

37. Arai K, Jin G, Navaratna D, Lo EH. Brain angiogenesis in developmental and pathological processes: neurovascular injury and angiogenic recovery after stroke. FEBS J. 2009;276(17):4644-52.

38. Morancho A, Rosell A, García-Bonilla L, Montaner J. Metalloproteinase and stroke infarct size: role for anti-inflammatory treatment? Ann N Y Acad Sci. 2010;1207:123-33.

39. Clark AW, Krekoski CA, Bou SS, Chapman KR, Edwards DR. Increased gelatinase A (MMP-2) and gelatinase B (MMP-9) activities in human brain after focal ischemia. Neurosci Lett. 1997;238(1-2):53-6.

40. Anthony DC, Ferguson B, Matyzak MK, Miller KM, Esiri MM, Perry VH. Differential matrix metalloproteinase expression in cases of multiple sclerosis and stroke. Neuropathol Appl Neurobiol. 1997;23(5):406-15.

41. Montaner J, Alvarez-Sabín J, Molina C, Anglés A, Abilleira S, Arenillas J, et al. Matrix metalloproteinase expression after human cardioembolic stroke: temporal profile and relation to neurological impairment. Stroke. 2001;32(8):1759-66.
42. Hayashi T, Kaneko Y, Yu S, Bae E, Stahl CE, Kawase T, et al. Quantitative analyses of matrix metalloproteinase activity after traumatic brain injury in adult rats. Brain Res. 2009;1280:172-7.

43. Ding JY, Kreipke CW, Schafer P, Schafer S, Speirs SL, Rafols JA. Synapse loss regulated by matrix metalloproteinases in traumatic brain injury is associated with hypoxia inducible factor-1alpha expression. Brain Res. 2009;1268:125-34.

44. Donkin JJ, Vink R. Mechanisms of cerebral edema in traumatic brain injury: therapeutic developments. Curr Opin Neurol. 2010;23(3):293-9. Review.

45. Vilalta A, Sahuquillo J, Rosell A, Poca MA, Riveiro $\mathrm{M}$, Montaner J. Moderate and severe traumatic brain injury induce early overexpression of systemic and brain gelatinases. Intensive Care Med. 2008;34(8):1384-92.

46. Wang X, Jung J, Asahi M, Chwang W, Russo L, Moskowitz $\mathrm{MA}$, et al. Effects of matrix metalloproteinase-9 gene knock-out on morphological and motor outcomes after traumatic brain injury. J Neurosci. 2000;20(18):7037-42.

47. Grossetete M, Phelps J, Arko L, Yonas H, Rosenberg GA. Elevation of matrix metalloproteinases 3 and 9 in cerebrospinal fluid and blood in patients with severe traumatic brain injury. Neurosurgery. 2009;65(4):702-8.

48. Asahi M, Asahi K, Jung JC, del Zoppo GJ, Fini ME, Lo EH. Role of matrix metalloproteinase 9 after focal cerebral ischemia: effects of gene knockout and enzyme inhibition with BB-94. J Cereb Blood Flow Metab. 2000;20(12):1681-9.

49. Suehiro E, Fujisawa H, Akimura T, Ishihara H, Kajiwara $\mathrm{K}$, Kato $\mathrm{S}$, et al. Increased matrix metalloproteinase-9 in blood in association with activation of interleukin- 6 after traumatic brain injury: influence of hypothermic therapy. J Neurotrauma. 2004;21(12):1706-11.

50. Rosenberg GA, Estrada EY, Dencoff JE. Matrix metalloproteinases and TIMPs are associated with bloodbrain barrier opening after reperfusion in rat brain. Stroke. 1998;29(10):2189-95.

51. Konopacki FA, Rylski M, Wilczek E, Amborska R, Detka D, Kaczmarek L, Wilczynski GM. Synaptic localization of seizure-induced matrix metalloproteinase-9 mRNA. Neuroscience. 2007;150(1):31-9.

52. Takács E, Nyilas R, Szepesi Z, Baracskay P, Karlsen B, Røsvold T, et al. Matrix metalloproteinase-9 activity increased by two different types of epileptic seizures that do not induce neuronal death: a possible role in homeostatic synaptic plasticity. Neurochem Int. 2010;56(6-7):799-809.

53. Suenaga $N$, Ichiyama T, Kubota M, Isumi $H$, Tohyama J, Furukawa S. Roles of matrix metalloproteinase-9 and tissue inhibitors of metalloproteinases 1 in acute encephalopathy following prolonged febrile seizures. J Neurol Sci. 2008;266(1-2):126-30. 